\title{
Starting Tutorials in headache medicine education
}

There is an obvious demand for education in headache medicine, in a scientific world advancing at an exponential rate. Both the fields of headache and craniofacial pain must follow this rule. This kind of education is essential for sector experts, neophytes, clinicians and general practitioners. The inflexibility of computer-based learning procedures and the lack of work covering overlapped medical specialties, such as headache, have led us to launch a series of Tutorials, in order to accomplish the following aims.

The term Tutorial represents an opportunity to develop skills in headache diagnosis and treatment. All of us agreed that clinical ability in headache medicine is mainly based on personal skills. Tutorials will help in the understanding of some of the basic concepts, which are sometimes far from daily clinical activity.

A pioneering systematic project concerning this important aspect of headache education has been carried out by Michael Björn Russell [1, 2]. From now on, we will promote this educational tool on each regular issue.

Last year, in an excellent pilot Tutorial by Tom Walley, attention was already focused on the understanding of basic pharmacoeconomics concepts applied to migraine [3]. His guidance into measurement of med- ical interventions in terms of costs (direct, indirect, intangible) and benefits, quantified according to both clinical and utility units, the starting point of any therapy evaluation, reached the final teaching aim.

A Tutorial by Peer Tfelt-Hansen appears in this issue, a useful guide for the critical reading of clinical trials [4]. The acknowledged expertise of the Author in this field, together with a simplification to make intelligible this otherwise not easily accessible topic, will especially facilitate the clinical readership in understanding the progress of a trial, instead of passively absorbing its conclusions.

In the next issue, attention will be dedicated to clinical epidemiology's base concepts [5]. This topic, clearly explained by Lars Jacob Stovner, can support the readership in surfing the waves of methodological issues, starting with epidemiological studies and so on. Finally, an approach maybe critical but never hostile - to the analysis of a new clinical headache form has been made by Fabio Antonaci, concerning the best diagnostic way to define a difficultto-treat headache entity, such as cervicogenic headache [6]. We must pay attention to the fact that patients suffering from "cervical head pain" could, if clinically seen with scotoma, easily fall into rather unortho- 
dox therapeutic plans. These two articles can currently be downloaded at the Online First section.

In the near future, other topics will be treated. Worth mentioning is a Tutorial concerning the role of placebo in headache management.

The aim of The Journal of Headache and Pain is to offer, through these Tutorials, an update for its readership regarding base concepts often borrowed from other disci- plines, in order to improve the cultural baggage of our young researchers and clinicians through a critical analysis of the contents that a scientific journal nowadays is able to offer.

\section{P. Martelletti}

Department of Medical Sciences, University la Sapienza, 2nd School of Medicine, Rome, Italy

e-mail: paolo.martelletti@uniromal.it

\section{References}

1. Russell MB (2000) Headache tutorial. Stud Health Technol Inform 78:207-212

2. Russell MB, Dremstrup Nielsen K, Rasmussen C, Schoenen J, Paiva T (2000) Multimedia education in headache: the European Neurological Network. Eur J Neurol 7:355-362
3. Walley T (2005) Pharmacoeconomics for migraine and headache researchers: basic concepts, methods and terminology. J Headache Pain 5:217-223

4. Tfelt-Hansen $P$ (2006) Understanding clinical trials in migraine. $J$ Headache Pain 7:101-108
5. Stovner LJ (2006) Headache epidemiology: how and why? J Headache Pain 7:DOI 10.1007/s10194-006-0276-4

6. Antonaci F, Bono G, Chimento F (2006) Diagnosing cervicogenic headache. J Headache Pain 7:DOI 10.1007/s10194-006-0277-3 